\title{
Reflections of an Earliest Established Screening and Testing Facility for COVID-19 in the Largest Metropolis, Pakistan
}

\author{
Fatima Kanwal ${ }^{1 *}$, Karishma Kanhya Lal ${ }^{1}$ and Asra Qureshi ${ }^{1}$ \\ ${ }^{1}$ Department of Family Medicine, Aga Khan University, Karachi, Pakistan
}

\begin{abstract}
As the world struggles to deal with the COVID-19 pandemic, this article talks about the experience and findings of the first COVID-19 screening facility in a tertiary care hospital in Karachi, Pakistan. Department of Family Medicine at Aga Khan University (AKU) took initiative where COVID-19 screening of stable patients first began in a dedicated area. Since testing kits were limited and community spread had not started, nurses would screen every walk-in patient based on the presence of fever, cough, travel history and exposure to COVID-19 positive patient in the last 14 days, and advise swab testing if indicated. Out of 742 -screened patients, approximately $66 \%$ were males and $34 \%$ were females. $38 \%$ of patients reported fever and $54 \%$ of patients reported cough. $8 \%$ (65) patients checked all three screening criteria of cough, fever and international travel exposure whereas $35 \%$ were asymptomatic. The majority had clinical exposure $(38.3 \%)$ followed by public exposure $(33.4 \%)$ and international travel exposure $(19.8 \%)$.
\end{abstract}

Keywords: COVID-19, COVID Pakistan, screening, ARDS, coronavirus, pandemic.

\section{INTRODUCTION}

The novel coronavirus started in Wuhan city of the Hubei province in China in December 2019. Soon after the first case was reported, the spread of the virus occurred through the different populations at varying speeds. Human to human transmission of SARS-Cov-2 through respiratory droplets was influenced by proximity, connectivity to Wuhan (China) as well as cultural and behavioral responses of the community, population density, etc. It has an incubation period of up to 2 weeks, after which the symptoms start to appear [1]. Many times, a person may be asymptomatic and still unknowingly be the source of transmission of SARS-Cov-2. As the spread has occurred at alarming rates, most countries did not have any time to prepare themselves for this global event. The WHO declared it pandemic on March 11 $1^{\text {th }}, 2020$ [2], when it had spread to more than 200 countries. As the experience of a worldwide pandemic is new, many countries had to take harsher measures and dedicate more to health resources to come up with testing methods for this infection. A university student returning from Iran was the first COVID-19 patient identified in Pakistan on $26^{\text {th }}$ February 2020 [3] as he tested positive at Aga Khan University Hospital (AKUH). A wave of panic hit people. Everyone who had been traveling or had the suspicion of being exposed wanted to be screened. The screening process had to be rapid, making use of limited resources. Hereby, we bring to you, such an experience of a healthcare screening set-up established on ad hoc basis and share the preliminary screening data so as to

\footnotetext{
*Corresponding author: Fatima Kanwal, Department of Family Medicine, Aga Khan University, Karachi, Pakistan,

Email:fatima.kanwal@aku.edu

Received: March 16, 2021; Revised: May 09, 2021; Accepted: May 31, 2021 DOI: https://doi.org/10.37184/lnjpc.2707-3521.3.5
}

further improve the screening and testing process by The Aga Khan University Hospital, a tertiary care set up in Karachi, Pakistan.

\section{MATERIALS AND METHODS}

Aga Khan University Hospital was the first private hospital in Pakistan to develop a COVID screening facility. A separate area was designated for the COVID screening of patients in the Community Health Center $(\mathrm{CHC})$ by the Department of Family Medicine in AKUH main campus. Since local transmission had not started and kits were limited [4], working on screening algorithm was started, based on current best available evidence. Data was collected from the $\mathrm{CHC}$ screening site from $11^{\text {th }}$ March to $22^{\text {nd }}$ March, 2020. Initially, screening was based upon symptoms of fever and cough in the last 14 days, international travel history and high-risk individuals such as health care workers and direct contacts of confirmed COVID patients. Nurses would screen every walk-in patient and advice swab testing if indicated. Family physicians performed a sampling of stable patients in a dedicated negative pressure room. All the screening data were maintained in a paper-based log sheet. SPSS version 22 was used for data analysis.

\section{RESULTS AND DISCUSSION}

In data of 742-screened patients, $65.6 \%$ were males and $34.4 \%$ were females. $38 \%$ of patients reported fever and $54 \%$ of the patients reported cough in the last 14 days. $65 \%$ of incoming people had one or more symptoms whereas approximately $35 \%$ were asymptomatic. $8 \%$ (65) patients checked all three screening criteria of cough, fever and international travel exposure. The gender-wise distribution of symptoms is 
shown in Table 1. Proportions of different categories of exposure are shown in Fig. (1).

Table 1: Gender-wise distribution of symptoms in patients coming for COVID-19 Screening.

\begin{tabular}{l|c|c}
\hline Symptom Evaluation & Male $(\mathbf{n}=\mathbf{4 8 7})$ & Female $(\mathbf{n = 2 5 5 )}$ \\
\hline Fever & $188(38.6 \%)$ & $97(38 \%)$ \\
\hline Cough & $262(53.8 \%)$ & $146(57.3 \%)$ \\
\hline Asymptomatic & $173(35.5 \%)$ & $87(34.1 \%)$ \\
\hline
\end{tabular}

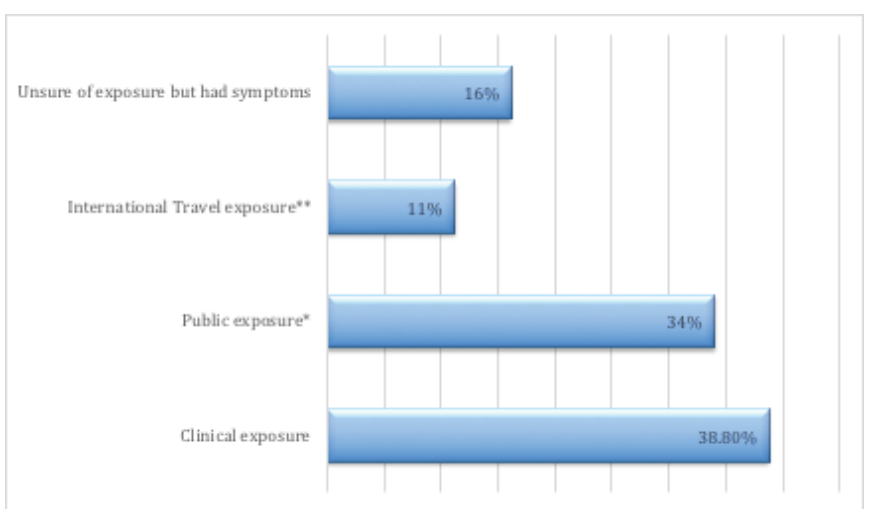

Fig. (1): Proportion of different exposure categories amongst screened cases at $\mathrm{CHC}$ facility of the Aga Khan University Hospital.

* International travel exposure either by themselves or by close family members.

** Public exposure such as receptionists and shopkeepers etc.

Pakistan was hit after the virus had spread to too many countries already. The hardest hits of which were in China, Italy, France, the UK, and the USA [5]. Hereby if we compare our data to China's initial screening data, where 135 patients were screened between January and February of 2020. Out of these 135 people, 59 $(44 \%)$ of them were males and $76(56 \%)$ were females. $111(82 \%)$ of those people had presented with a travel history and symptoms of fever, cough, sore throat, or shortness of breath. Around $11(8 \%)$ people had no symptoms but a travel history in countries outside China. Additionally, $13(10 \%)$ people came with no symptoms or travel history, just for reassurance [6].

What we learned from this experience is that the element of anxiety might be one of the major causes of asymptomatic patients coming for screening. Researchers have reported public education in a timely manner with correct information from authentic sources as a core measure to deal with current pandemic anxiety $[7,8]$. Mass education via social and other media, COVID helplines, and developing COVID screening apps for employees are some of the initiatives that are taken AKU in order to calm down and appropriately manage COVID anxiety. In our study, a history of exposure to people with COVID-19 was also accounted for, making it one of the reasons why more people were concerned.
Followed by this experience, screening became more stringent as asymptomatic carriers are a great threat. We also learned that any other new-onset symptoms during the incubation period should be asked for as it's a new disease and other presenting features may become identifiable over time.

However, because screening can miss people who are infected, public health measures such as face coverings, physical distancing, and quarantine for those who are apparently healthy, continue to be very important.

Learning from this experience, risk assessment of exposure and symptoms is started with regular revision to incorporate any updated changes. Rigorous infection control protocols, evidence-based and safe care for infected patients, advising government and other hospitals and health care professionals, initiation of telemedicine clinics, are few salient features of AKU leading this pandemic from the front. Currently, in AKU, many pieces of research are underway under the umbrella of the COVID-19 Research Coordinating Unit as well as collaboration with WHO on the research front is ongoing [9].

\section{RECOMMENDATION}

As pandemic continues to unfold, and social restrictions are eased, more people are becoming infected; health care workers remain a high-risk population so availability and proper use of PPEs should be made a priority. Timely awareness of the population with correct information in a sensitive manner should be arranged. In order to improve the screening process, it is recommended to dedicate a separate area, wherever possible till the behavior of the disease is better understood. Prior training of screening staff, inquiring of symptoms other than known ones along with evidence-based risk assessment is advisable to better allocate available resources. In the future, a risk assessment should also be linked with the actual disease status of patients screened so the risk assessment tool can be validated for future use. Forethoughtful constant vigilance and research is the key to keep this pandemic in check. After all, it is a new virus, and screening is one of the first steps to know more and do more.

\section{CONFLICT OF INTEREST}

The authors declare no conflict of interest.

\section{ACKNOWLEDGEMENTS}

The authors acknowledge Department Chair Dr. Unab Khan for permitting write-up and the nursing staff who screened patients and collected data. 


\section{REFERENCES}

1. Thevarajan I, Buising KL, Cowie BC. Clinical presentation and management of COVID-19. Med J Aust 2020; 213(3): 134-9.

2. Cucinotta D, Vanelli M. WHO declares COVID-19 a pandemic. Acta Bio Medica: Atenei Parmensis 2020; 91(1): 157.

3. Abid K, Bari YA, Younas M, Tahir Javaid S, Imran A. < ? covid19?> Progress of COVID-19 Epidemic in Pakistan. Asia Pac J Public Health 2020; 32(4): 154-6.

4. Khanain w. Pakistan now equipped to test for novel coronavirus, says PM's aide. DAWN. 2020 feburary 22020.

5. WHO. Coronavirus disease 2019 (COVID-19) Situation Report 71. 202031 March 2020. Report No.
6. Lin M, Beliavsky A, Katz K, Powis JE, Ng W, Williams V, et al. What can early Canadian experience screening for COVID-19 teach us about how to prepare for a pandemic? CMAJ 2020; 192(12): E314-E8.

7. Roy D, Tripathy S, Kar SK, Sharma N, Verma SK, Kaushal V. Study of knowledge, attitude, anxiety \& perceived mental healthcare need in Indian population during COVID-19 pandemic. Asian J Psychiatr 2020; 51: 102083.

8. Shanafelt T, Ripp J, Trockel M. Understanding and addressing sources of anxiety among health care professionals during the COVID-19 pandemic. Jama 2020; 323(21): 2133-4.

9. University TAK. AKU's Response to the COVID-19 Pandemic in Pakistan 2020 [updated July 28,2020; cited 2021 15-3]. Available from: https://www.aku.edu/Pages/covid-19-aku-response.aspx\#2. 